\title{
Posterior Lumbar Interbody Fusion with Spinal Decompression using Minimally Invasive Spine Surgery in the Treatment of Symptomatic Degenerative Spinal Stenosis
}

\author{
Michael Rothmans Immanuel Silaban ${ }^{1 *}$, Pranajaya Dharma Kadar ${ }^{2}$ \\ ${ }^{1}$ Department of General Medicine, Faculty of Medicine, Universitas Sumatera Utara, North Sumatera, Indonesia; ${ }^{2}$ Department \\ of Orthopaedic and Traumatology, Faculty of Medicine, Universitas Sumatera Utara, North Sumatera, Indonesia
}

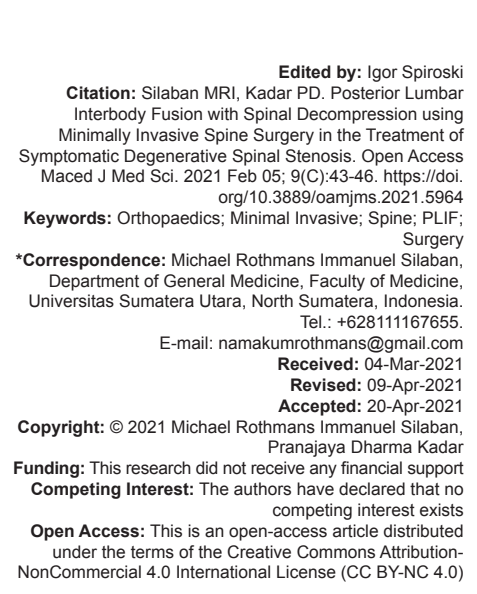

Abstract

BACKGROUND: Lumbar spinal stenosis occurs predominantly in elderly person as a degenerative progress. It could be acquired such as spondylosis, spondylolisthesis and trauma. Signs and symptoms of lumbar spinal stenosis are low back pain, sciatica, neurogenic intermittent claudication, motor and sensory deficits and reflex changes. Aching spreads over the lumbosacral dermatomes or painful parasthesias can sometime develop. These symptoms may be triggered by walking and producing flexion motion of the lumbar spine such as sitting or crouching forwards and relieved by resting for several minutes. Lumbar spinal stenosis is often the result of advanced degeneration of motion segments of the lumbar spine. Loss of disc height, facet displacement and hypertrophy, spondylosis, and spondylolisthesis, all contribute to impact the spinal canal and intervertebral foramen in lumbar stenosis. Using a minimal invasive surgery (MIS) make a better postoperative condition, provides benefits such as earlier mobilization, shorter hospital stay, reduced hospital costs, and morbidity reduction.

CASE PRESENTATION: We present a 67-year-old woman with low back pain that has occurred for 2 years and had been worsening for the past 3 months. She described the pain as an intermittent ache down on her leg, the pain was severe and worsened when the patient is standing, sitting, or walking for a long time. The pain severity was measured by Visual Analog Scale (VAS), graded between $7 / 10$ and $9 / 10$. She also complained numbness from the bilateral gluteal region to the lateral side of lower extremities, when she feels too tired. After the examination, the patient agreed to undergo the suggested operative procedure and gave the consent at the hospital.

CONCLUSION: Minimally invasive spine surgery using posterior lumbar interbody fusion can be recommended in our opinion for symptomatic degenerative spinal stenosis with segmental instability because the benefits such as lower back pain relief, improvement of the motoric \& sensoric function, early mobilization compared to classic PLIF technique.

\section{Introduction}

Lumbar spinal stenosis is often the result of advanced degeneration of motion segments of the lumbar spine. The incidence of this case is $3.57 \%$ (3570/100,000) population in Southeast Asia [1]. The main symptoms are low back pain, numbness, and weakness in the lower extremity that occur and intensify on walking caused by the load of the body weight on the spine. Loss of disc height, facet displacement and hypertrophy, spondylosis, and spondylolisthesis, all contribute to impact the spinal canal and intervertebral foramen in lumbar stenosis [2]. There is a subgroup of patients with spinal stenosis in whom the spine is unstable preoperatively or become destabilized following decompression who would benefit from fusion procedure. In some situations, other diagnoses can also lend themselves to the posterior lumbar interbody fusion technique, such as in patients who have already been operated on through an anterior approach [2], [3].

\section{Objective}

Surgical treatment of lumbar spinal stenosis by posterior lumbar interbody fusion is indicated for patients with symptoms of low back pain and lower limb radicular pain that are unsuccessful treated with medicines and/ or patients with persisting or worsening neurological deficit. However, this procedure may lead to possible complications. This case report study was conducted to show how our hospital handling spinal stenosis case.

\section{Case Report}

A 67-year-old woman presented with low back pain that has occurred for 2 years and had been worsening for the past 3 months. She described the pain as an intermittent ache down on her leg, the pain was severe and worsened when the patient is standing, sitting, or walking for a long time. The pain severity was measured 
by Visual Analog Scale (VAS), graded between 7/10 and $9 / 10$. She also complained numbness from the bilateral gluteal region to the lateral side of lower extremities, when she feels too tired. On the physical examination, she was found to have limited range of motion for flexion and extension, on the lumbar spine, due to pain. The motoric strength of both lower limbs was decreased to $2 / 5$, so did the sensory function such as thick feeling, numb, and decreased temperature sensation from the lateral cruris to medial area of foot. The radiological examination showed a severe stenosis at lumbar spinal bone region on L4-L5 area (Figure 1). After the examination, the patient agreed to undergo the suggested operative procedure and gave the consent at the hospital.

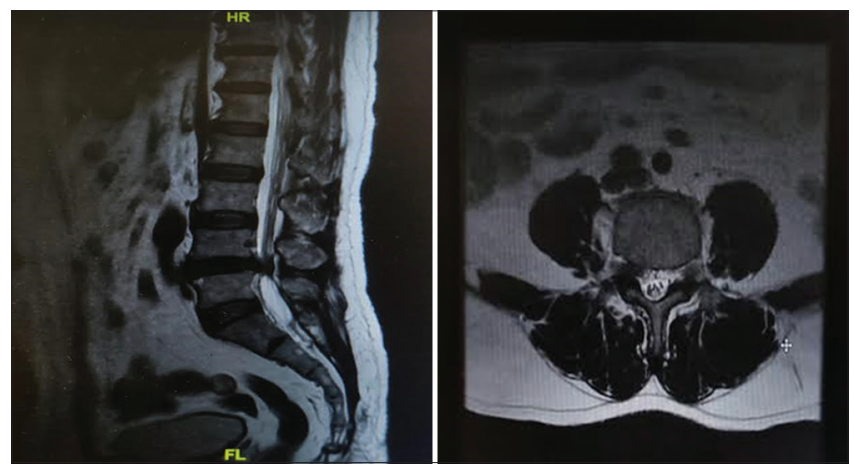

Figure 1: Pre-operative MRI examination shown L4-L5 stenosis

\section{Methods}

\section{Surgical technique}

The patient was placed under general anesthesia in the prone position. With the muscles adjacent to the spine retraced laterally to minimize damage of medulla spinalis, the lateral area of the lamina and the posterior joint was exposed through posteromedial approach, while the transverse process was not exposed. Nerve root decompression was achieved by performing laminectomy, complete excision of the inferior articular process, and discectomy using posterior lumbar interbody fusion (PLIF) technique.

The insertion of a single cage was attached on the side with the more severe symptoms or the more severe stenotic foramen, and the duramater and the nerve root were medially retracted. Extensive removal of the intervertebral disc and the adjacent end plates was performed on the ipsilateral side with using a pituitary Rongeur and a curved curette until the subchondral bone was exposed.

The size of a cage was determined based on the disc height. The involved titanium cages were used and they were rectangular in shape and radiopaque on the X-ray examination. Pedicle screw fixation was carried out after inserting the cage to secure the stability and to improve the bony union immediately after surgery. Standard wound closure was performed following hemostasis.

\section{Results}

No complications occurred during or after the surgery. Patients were given IV ketorolac as the analgesic post-operative. Four days after the surgery, her low back pain and claudication intermittent were decreased. It was proved by decrease in the VAS, became $4 / 10$ and $6 / 10$. Motor response and sensory function were improved. After the surgery, the motoric strength becomes $4 / 5$ and the patient can respond to sensory stimuli. The radiology examination was immediately taken after the surgery and was compared to the previous X-ray. It was found that the intervertebral disc backs to the normal height (Figure 2). The X-ray was performed on the anteroposterior and lateral position. The position of cage and the pedicle screw stability was also evaluated after the surgery by the X-ray examination. The patient had a follow-up 1 month and 3 months, to assess physical status, motoric and sensory function to evaluate the functional outcome after the surgery and the result was normal, the patient does not have any sequelae (Figure 3 ).

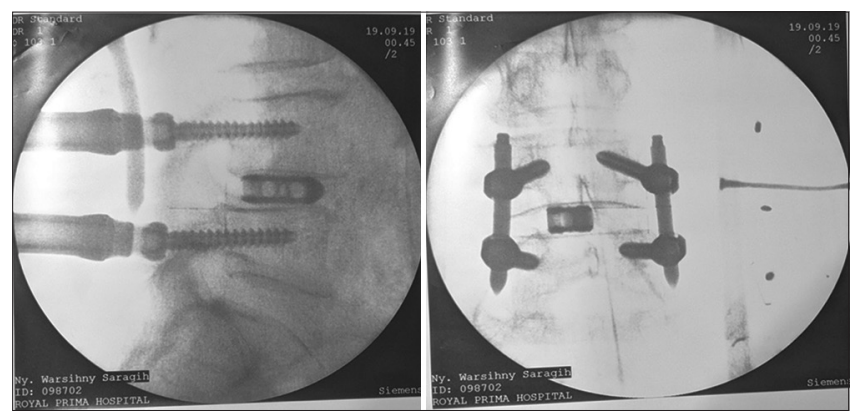

Figure 2: A 67-year-old female with lumbar spinal stenosis at L4-L5 had a posterior lumbar interbody fusion surgery technique with cage and pedicle screw fixation

\section{Discussion}

Lumbar spinal stenosis occurs predominantly in elderly person as a degenerative progress. It could be acquired such as spondylosis, spondylolisthesis, and trauma. Signs and symptoms of lumbar spinal stenosis are low back pain, sciatica, neurogenic intermittent claudication, motor and sensory deficits, and reflex changes [3], [4]. Aching spreads over the lumbosacral dermatomes or painful paresthesias can sometime develop. These symptoms may be triggered by walking and producing flexion motion of the lumbar spine such as sitting or crouching forwards and relieved by resting for several minutes. Because an improvement in low back pain in patients with lumbar spine stenosis and degenerative instability is very important, for patients with intolerable pain who fail conservative therapies and minimally invasive treatments, surgery is indicated [5]. Our patient had severe lumbar spinal 

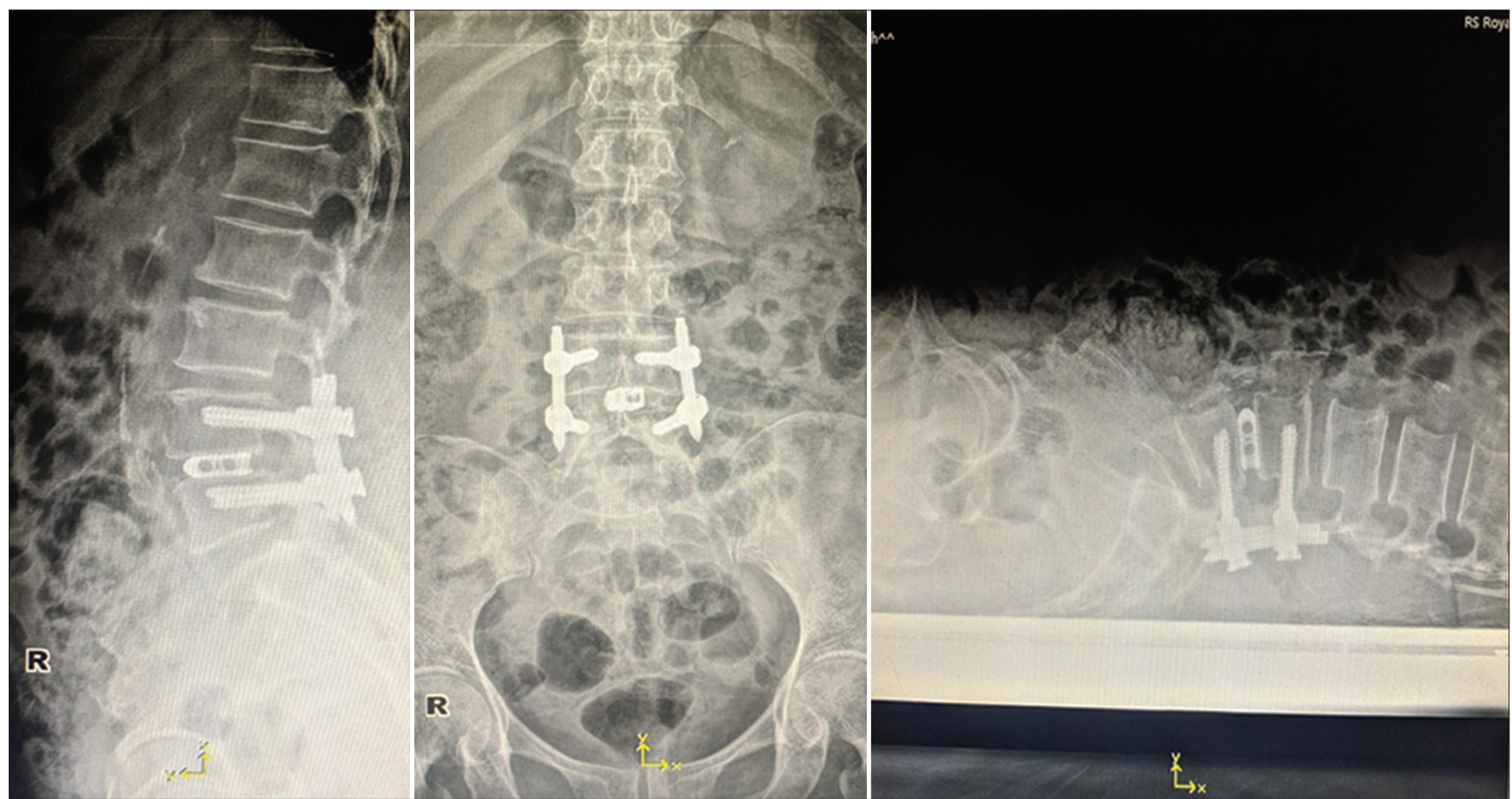

Figure 3: Post-operative $X$-rays show the restoration of the intervertebral disc height

stenosis demonstrated by radiological findings and does the minimal invasive surgery (MIS) surgery using a PLIF approach. Although the efficacy of fusion in degenerative disease remains controversial, several studies have recommended instrumentation to improve the fusion rate. Furthermore, spinal fusion may prevent recurrent stenosis [6].

Lumbar interbody fusion is a well-validated technique with several different approaches such as anterior, lateral, transforaminal, and posterior approaches for a variety of conditions requiring spine stabilization [6], [7]. Among them, PLIF is frequently used and may provide a higher immediate stability, especially in lateral bending. However, it may be limited by the thecal and nerve root retraction. Complications are nightmares for spine surgeons. The most common complications associated with PLIF are intraoperative neurologic injury, interbody implant or bone graft migration, dural tear, surgical site infection, and so on [8]. The overall reported complication rates about $8 \%$, such as neurological injuries of PLIF which did not include potential pseudoarthroses [9]. Implant migration may be uncommon but very tricky. Dural tear or cerebral fluid leakage is a common complication whether during classic PLIF compared to MIS, wide decompression, and fusion may be accompanied by substantial complications such as post-operative blood loss, a prolonged operation time, and postoperative infection. Further, long-segment fusion and abundant blood loss may increase the incidences of complications. Excessive bleeding often requiring multiple blood transfusions [10].

Using a MIS makes a better post-operative condition, provides benefits such as earlier mobilization, shorter hospital stay, reduced hospital costs, and morbidity reduction. Although the goal of treatment of spinal stenosis is to decompress the affected neural structure, it is difficult to reduce back pain and neurological symptoms by decompression without performing fusion surgery in the patients who have multilevel foraminal stenosis with back pain.

\section{Conclusion}

Minimally invasive spine surgery using posterior lumbar interbody fusion can be recommended in our opinion for symptomatic degenerative spinal stenosis with segmental instability because the benefits such as lower back pain relief, improvement of the motoric and sensory function, and early mobilization compared to classic PLIF technique. However, because the patients are elderly patient or above 65 years old, certainly many things that should be considered very carefully such as how the physical condition and whether there are other concomitant disease or comorbid factors that can complicate the surgical procedure.

\section{References}

1. Farrokhi MR, Yadollahikhales G, Gholami M, Mousavi SR, Mesbahi AR, Asadi-Pooya AA. Clinical outcomes of posterolateral fusion vs. posterior lumbar interbody fusion in patients with lumbar spinal stenosis and degenerative instability. 
Pain Physician. 2018;21(4):383-406. https://doi.org/10.36076/ ppj.2018.4.383

PMid:30045595

2. Kim DH, Jeong ST, Lee SS. Posterior lumbar interbody fusion using a unilateral single cage and a local morselized bone graft in the degenerative lumbar spine. Clin Orthop Surg. 2009;1(4):214-21. https://doi.org/10.4055/cios.2009.1.4.214

PMid:19956479

3. Tomita K. Diagnosis and treatment of lumbar spinal canal stenosis. J Jpn Med Assoc. 2012;128:1790-4.

4. Choi JM, Choi MK, Kim SB. Perioperative results and complications after posterior lumbar interbody fusion for spinal stenosis in geriatric patients over than 70 years old. J Korean Neurosurg Soc. 2017;60(6):684-90. https://doi.org/10.3340/ jkns.2017.0203

PMid:29142628

5. Liang L, Jiang WM, Li XF, Wang $\mathrm{H}$. Effect of fusion following decompression for lumbar spinal stenosis: A meta-analysis and systematic review. Int J Clin Exp Med. 2015;8(9):14615-24. PMid:26628944

6. Mobbs RJ, Phan K, Malham G, Seex K, Rao PJ. Lumbar interbody fusion: Techniques, indications and comparison of interbody fusion options including PLIF, TLIF, MI-TLIF, OLIF/ ATP, LLIF and ALIF. J Spine Surg. 2015;1(1):2-18. https://doi. org/10.28962/01.3.072

PMid:27683674

7. Dharma AS, Ermawan R, Utomo P, Handojo HT. Correlation of fusion rate on lumbar spinal stenosis post PLIF with functional outcome. Biomedika. 2009;11(2):61-7. https://doi.org/10.23917/ biomedika.v11i2.7613

8. Fenton-White HA. Trailblazing: The historical development of the posterior lumbarinterbody fusion (PLIF). Spine J. 2021;10(2):15299127-3. https://doi.org/10.1016/j.spinee.2021.03.016 PMid:33757870

9. Yang LH, Liu W, Li J, Zhu WY, An LK, Yuan S, et al. Lumbar decompression and lumbar interbody fusion in the treatment of lumbar spinal stenosis: A systematic review and metaanalysis. Medicine (Baltimore). 2020;99(27):e20323. https://doi.org/10.1097/md.0000000000020323 PMid:32629626

10. Lam K, Al-Mousa A, Manson N. Do outcomes vary following minimally invasive lumbar interbody fusion (MILIF) in patients with spinal stenosis? A multi-centre subgroup analysis study. Spine J. 2016;16(4):S47. https://doi.org/10.1016/j. spinee.2016.01.032 